TRANSACTIONS OF THE

AMERICAN MATHEMATICAL SOCIETY

Volume 355, Number 4, Pages 1491-1503

S 0002-9947(02)03178-1

Article electronically published on December 4, 2002

\title{
SOME TWO-STEP AND THREE-STEP NILPOTENT LIE GROUPS WITH SMALL AUTOMORPHISM GROUPS
}

\author{
S. G. DANI
}

\begin{abstract}
We construct examples of two-step and three-step nilpotent Lie groups whose automorphism groups are "small" in the sense of either not having a dense orbit for the action on the Lie group, or being nilpotent (the latter being stronger). From the results we also get new examples of compact manifolds covered by two-step simply connected nilpotent Lie groups which do not admit Anosov automorphisms.
\end{abstract}

Let $G$ be a connected Lie group and Aut $(G)$ the group of all (continuous) automorphisms of $G$. When does the action of Aut $(G)$ on $G$ have a dense orbit? It turns out that if this holds, then $G$ is a nilpotent Lie group (see 4]). However, it does not hold for all nilpotent Lie groups, and a characterization of the class of groups for which it holds seems to be a remote possibility. When $G$ is a vector space $\operatorname{Aut}(G)$ is its general linear group, and the action has an open dense orbit, namely the complement of the zero. On the other hand, there exist 3-step simply connected nilpotent Lie groups such that every orbit of the action is a proper closed subset; this holds for the simply connected Lie group corresponding to the Lie algebra described in [9]; see also $\S$ below. Among the simply connected Lie groups this brings us to considering the question for 2-step nilpotent Lie groups. In this note, using a representation of $P S L(2, \mathbb{R})$, we construct an example of a 2-step simply connected nilpotent Lie group $G$ for which the action of the automorphism group has no dense orbit; see Theorem 2.1

We discuss the question also for Lie groups which are not simply connected, and show that while for all connected abelian groups other than the circle the automorphism group action has dense orbits, among the quotients of the group $G$ as above by discrete central subgroups there are nilpotent Lie groups $G^{\prime}$ such that every orbit of Aut $\left(G^{\prime}\right)$ on $G^{\prime}$ consists of either one or two cosets of the commutator subgroup $\left[G^{\prime}, G^{\prime}\right]$, the latter being a closed subgroup; see Corollary 5.2

The method involved also enables us to give examples of 3-step simply connected nilpotent Lie groups whose automorphism groups are nilpotent and act on the Lie algebras by unipotent transformations; this condition implies in particular that all orbits of the actions are closed; see $\S 4$ An example of a 6-step simply connected nilpotent Lie group with this property was given earlier in 10.

Another motivation for studying the automorphism groups of nilpotent Lie groups comes from the question of understanding which compact nilmanifolds support Anosov diffeomorphisms and which do not. It is known that if $G$ is a free $k$-step nilpotent Lie group over an $n$-dimensional vector space $V$ with $k<n$, then

Received by the editors April 29, 2002 and, in revised form, July 12, 2002.

2000 Mathematics Subject Classification. Primary 22D45, 22E25; Secondary 22D40, 37D20. 
for any lattice $\Gamma$ in $G$ the nilmanifold $G / \Gamma$ admits Anosov automorphisms; see [3], p. 558; see [7] for another approach to the question. There are also other classes of compact nilmanifolds for which this holds; see [1], [3], [7], [8]. However, in general, compact nilmanifolds need not admit Anosov automorphisms. Clearly, if $G$ is a 2 -step simply connected nilpotent Lie group such that $[G, G]$ is one-dimensional, then $G / \Gamma$ cannot admit an Anosov automorphism, for any lattice $\Gamma$. Only a few other examples of 2-step simply connected nilpotent Lie groups are known with this property; see [1], 7], [12]. We shall show that for the 2-step nilpotent Lie group $G$ in our example, if $\Gamma$ is a lattice in $G$, then the nilmanifold $G / \Gamma$ has no Anosov automorphism, and also no ergodic automorphism; see Corollary 6.1

\section{Lie ALGEBRAS AND LiE GROUpS}

We recall that a Lie algebra $\mathcal{G}$ is said to be nilpotent if the central series $\left\{\mathcal{G}_{i}\right\}$ defined by $\mathcal{G}_{0}=\mathcal{G}$ and $\mathcal{G}_{i+1}=\left[\mathcal{G}, \mathcal{G}_{i}\right]$ for all $i \geq 0$ terminates; namely, there exists $k \geq 1$ such that $\mathcal{G}_{k}=0$. If $k$ is the smallest integer for which this holds, then $\mathcal{G}$ is said to be $k$-step nilpotent. We say that a connected Lie group $G$ is a $k$-step nilpotent Lie group if the Lie algebra of $G$ is a $k$-step nilpotent Lie algebra.

For a Lie group $G$ we shall denote by $\operatorname{Aut}(G)$ the group of all continuous automorphisms of $G$, and similarly for a Lie algebra $\mathcal{G}$ we denote by $\operatorname{Aut}(\mathcal{G})$ the group of all Lie automorphisms of $\mathcal{G}$.

Let $G$ be a connected nilpotent Lie group. Let $G^{(1)}=\overline{[G, G]}$, and let $Z(G)$ denote the center of $G$. Suppose that $Z(G)$ is contained in $G^{(1)}$; this condition holds for the nilpotent groups discussed in the following sections. Then for any (continuous) homomorphism $\psi: G / G^{(1)} \rightarrow Z(G)$ the map $\tau: G \rightarrow G$ defined by $\tau(g)=g \psi\left(g G^{(1)}\right)$ for all $g \in G$ is an automorphism of $G$; we shall call an automorphism arising in this way a shear automorphism. The class of all shear automorphisms forms a normal subgroup of Aut $(G)$. Every automorphism $\tau$ of $G$ factors to an automorphism of $G / G^{(1)}$; we denote the factor of $\tau$ on $G / G^{(1)}$ by $\bar{\tau}$. Clearly $\tau \mapsto \bar{\tau}$ is a homomorphism of Aut $(G)$ into Aut $\left(G / G^{(1)}\right)$. We denote by $\mathcal{A}(G)$ the image of the homomorphism, namely,

$$
\mathcal{A}(G)=\left\{\bar{\tau} \in \operatorname{Aut}\left(G / G^{(1)}\right) \mid \tau \in \operatorname{Aut}(G)\right\} .
$$

We note that for all shear automorphisms, as well as all inner automorphisms of $G$, the factor on $G / G^{(1)}$ is trivial.

Suppose now that $G$ is a simply connected 2-step nilpotent Lie group, and $\mathcal{G}$ is the Lie algebra of $G$. Then $G^{(1)}=[G, G]$, namely $[G, G]$ is closed, and $G /[G, G]$ and $[G, G]$ are (topologically isomorphic to) vector spaces; they may be identified canonically with $\mathcal{G} /[\mathcal{G}, \mathcal{G}]$ and $[\mathcal{G}, \mathcal{G}]$ respectively. Also in this case $\operatorname{Aut}(G)$ can be realized as Aut $(\mathcal{G})$, by identifying each automorphism with its derivative on $\mathcal{G}$.

We recall also the following well-known fact (see [6], Lemma 3.1, for a proof).

Lemma 1.1. Let $G$ be a simply connected nilpotent Lie group and $\mathcal{G}$ be the Lie algebra of $G$. Let $\tau \in \operatorname{Aut}(\mathcal{G})$ and suppose that $\bar{\tau}$ is unipotent (as an element of $G L(\mathcal{G} /[\mathcal{G}, \mathcal{G}])$ ). Then $\tau$ is unipotent (as an element of $G L(\mathcal{G})$ ).

Now let $V$ be a (finite-dimensional) vector space and let $W$ be a subspace of $\wedge^{2} V$, the second exterior power of $V$. We can associate to these, canonically, a 2-step nilpotent Lie group as follows. Let $V^{\prime}=\left(\wedge^{2} V\right) / W$ and $\mathcal{G}=V \oplus V^{\prime}$. We set $\left[v_{1}, v_{2}\right]=v_{1} \wedge v_{2} \bmod W$ for all $v_{1}, v_{2} \in V$, and $[x, y]=0$ for all $x \in \mathcal{G}$ and $y \in V^{\prime}$. 
These relations extend uniquely to a Lie bracket operation on $\mathcal{G}$. Furthermore, $\mathcal{G}$ is a 2-step nilpotent Lie algebra, with $\mathcal{G} /[\mathcal{G}, \mathcal{G}]=V$. This construction is nothing but the construction of a 2-step nilpotent Lie algebra as a quotient of a free 2-step nilpotent Lie algebra (see [1], for instance). Conversely, every 2-step nilpotent Lie algebra $\mathcal{G}$ can be realized as a Lie algebra associated to a datum as above, with $V=\mathcal{G} /[\mathcal{G}, \mathcal{G}]$, and $W$ a suitable subspace of $\wedge^{2} V$. Let $G$ be the simply connected Lie group corresponding to the Lie algebra $\mathcal{G}$ associated to a pair $V, W$ as above. Then $G /[G, G]$ may be realized canonically as $V$; this identifies the subgroup $\mathcal{A}(G)$ with a subgroup of $G L(V)$. It is easy to see that $x \in G L(V)$ is of the form $\bar{\tau}$ for some $\tau \in \operatorname{Aut}(G)$ if and only if the subspace $W$ of $\wedge^{2} V$ is invariant under the action induced by $x$ on $\wedge^{2} V$. It follows in particular that $\mathcal{A}(G)$ is an algebraic subgroup of $G L(V)$.

We note also the following:

Proposition 1.2. Let $G$ be a simply connected nilpotent Lie group. Then every orbit of Aut $(G)$ on $G$ is open in its closure. In particular, every dense orbit is open. Consequently, if the $\mathcal{A}(G)$-action on $V$ has no open orbit, then the $\operatorname{Aut~}(G)$-action on $G$ has no dense orbit.

Proof. Let $\mathcal{G}$ be the Lie algebra of $G$. Then $\operatorname{Aut}(\mathcal{G})$ is an algebraic subgroup of $G L(\mathcal{G})$, and this implies that the orbits of its action on $\mathcal{G}$ are open in their closures (see [2]). Since $G$ is simply connected and nilpotent, the exponential map is a diffeomorphism of $\mathcal{G}$ onto $G$ and it is equivariant under the actions of the respective automorphism groups. Therefore for the action of Aut $(G)$ on $G$ also every orbit is open in its closure. The second assertion is immediate from the first. The last assertion follows from the fact that the $\mathcal{A}(G)$-action on $V$ is a factor of the $\operatorname{Aut}(G)$-action on $G$.

\section{A Representation}

In this section we describe a construction of a representation and prove some properties. The results will be used in the later sections to give examples of nilpotent Lie algebras.

Let $\mathcal{S}$ be the vector space of $3 \times 3$ symmetric matrices with real entries. For each $k, l=1,2,3$ we denote by $E_{k l}$ the $3 \times 3$ matrix in which the $(k, l)$-entry (in the $k$ th row and $l$ th column) is 1 and all other entries are 0 . Let $V$ be the subspace of $\mathcal{S}$ defined by

$$
V=\left\{\sum_{k, l=1}^{3} \sigma_{k l} E_{k l} \in \mathcal{S} \mid \sigma_{22}=\sigma_{13}+\sigma_{31}=2 \sigma_{13}\right\} .
$$

Let $\sigma_{1}, \ldots, \sigma_{5}$ be the elements defined as follows, forming a basis of $V$ :

$$
\begin{gathered}
\sigma_{1}=2 E_{11}, \sigma_{2}=E_{12}+E_{21}, \sigma_{3}=E_{13}+2 E_{22}+E_{31}, \\
\sigma_{4}=E_{23}+E_{32}, \text { and } \sigma_{5}=2 E_{33} .
\end{gathered}
$$

Let $W$ be the subspace of $\wedge^{2} V$ spanned by the three elements $\sigma_{1} \wedge \sigma_{4}-\sigma_{2} \wedge \sigma_{3}$, $\sigma_{1} \wedge \sigma_{5}-\sigma_{2} \wedge \sigma_{4}$ and $\sigma_{2} \wedge \sigma_{5}-\sigma_{3} \wedge \sigma_{4}$.

Let $\delta, \nu^{+}$and $\nu^{-}$be the matrices defined by

$$
\delta=\left(\begin{array}{ccc}
2 & 0 & 0 \\
0 & 0 & 0 \\
0 & 0 & -2
\end{array}\right), \nu^{+}=\left(\begin{array}{ccc}
0 & 1 & 0 \\
0 & 0 & 1 \\
0 & 0 & 0
\end{array}\right), \text { and } \nu^{-}=\left(\begin{array}{ccc}
0 & 0 & 0 \\
1 & 0 & 0 \\
0 & 1 & 0
\end{array}\right)
$$


The space spanned by these three matrices is a Lie subalgebra of the Lie algebra of $3 \times 3$ matrices of trace 0 , namely the Lie algebra of $S L(3, \mathbb{R})$. We denote this Lie subalgebra by $\mathcal{H}$. We note that it is isomorphic to the Lie algebra of $S L(2, \mathbb{R})$, with $\delta, \nu^{+}$and $\nu^{-}$corresponding to the standard basis of the latter; in particular, the subspace spanned by $\delta$ is a Cartan subalgebra. Let $H$ be the connected Lie subgroup of $S L(3, \mathbb{R})$ corresponding to $\mathcal{H}$. Consider the action of $H$ on $\mathbb{R}^{3}$ given by restriction of the natural action of $S L(3, \mathbb{R})$ on $\mathbb{R}^{3}$. We see that there is no proper nonzero subspace on $\mathbb{R}^{3}$ invariant under both $\exp \nu^{+}$and $\exp \nu^{-}$. It follows therefore that the action of $H$ on $\mathbb{R}^{3}$ is irreducible. Since $H$ contains an element, viz. $\exp \delta$, with distinct eigenvalues, this implies that every element of the center of $H$ acts by scalar multiplication by real numbers, and considering the determinant we see that it must be trivial. Thus $H$ has trivial center. This shows that $H$ is Lie isomorphic to $P S L(2, \mathbb{R})$, the adjoint group of $S L(2, \mathbb{R})$.

Consider the action of $S L(3, \mathbb{R})$ on $\mathcal{S}$ given by $(x, \sigma) \mapsto x \sigma x^{\mathrm{t}}$ for all $x \in S L(3, \mathbb{R})$ and $\sigma \in \mathcal{S}$, where $x^{\mathrm{t}}$ denotes the transpose of $x$. It is straightforward to verify that the subspace $V$ is invariant under the action of $H$ (obtained by restriction); it suffices to verify that $V$ is invariant under the corresponding action of the Lie algebra, given by $(\xi, \sigma) \mapsto \xi \sigma+\sigma \xi^{\mathrm{t}}$, for all $\xi \in \mathcal{H}$ and $\sigma \in \mathcal{S}$, and furthermore it is enough to consider $\xi=\delta, \nu^{+}$and $\nu^{-}$. The elements $\sigma_{1}, \ldots, \sigma_{5}$ are weight vectors with respect to $\delta$ with weights $4,2,0,-2$ and -4 respectively, and the latter being distinct shows that the representation of $H$ over $V$ is irreducible.

We shall realize $H$ as a subgroup of $G L(V)$ by identifying each $h$ in $H$ with its action on $V$ (this is indeed an injective correspondence). We denote by $D$ the one-parameter subgroup of $G L(V)$ corresponding to the one-parameter subgroup $\{\exp t \delta \mid t \in \mathbb{R}\}$. We note that for any nontrivial element of $D, \sigma_{1}, \ldots, \sigma_{5}$ are eigenvectors with distinct eigenvalues, and hence the centralizer of $D$ in $G L(V)$ consists of diagonal matrices with respect to the basis $\sigma_{1}, \ldots, \sigma_{5}$.

Let $\mathcal{A}$ be the subgroup consisting of all $x$ in $G L(V)$ such that $W$ is invariant under the action induced by $x$ on $\wedge^{2} V$. A straightforward computation shows that $H$ (viewed as a subgroup of $G L(V))$ is contained in $\mathcal{A}$. We note that $\mathcal{A}$ is an algebraic subgroup of $G L(V)$. Let $\mathcal{A}_{u}$ be the unipotent radical of $\mathcal{A}$, namely the largest normal subgroup consisting of unipotent elements. Then the set of common fixed points of the action of $\mathcal{A}_{u}$ on $V$ is a nonzero subspace invariant under $H$, and since the $H$-action on $V$ is irreducible, it follows that $\mathcal{A}_{u}$ fixes all points of $V$, which means that $\mathcal{A}_{u}$ is trivial. Therefore $\mathcal{A}$ is reductive.

Theorem 2.1. $\mathcal{A}=Z H$, where $Z$ is the subgroup of $G L(V)$ consisting of scalar multiplications by real numbers. The $\mathcal{A}$-action on $V$ has no open orbit.

Proof. Let $\mathcal{A}^{0}$ be the connected component of the identity in $\mathcal{A}$ and let $S=\left[\mathcal{A}^{0}, \mathcal{A}^{0}\right]$, the commutator subgroup. Since $\mathcal{A}^{0}$ is reductive, it follows that $S$ is a semisimple subgroup of $G L(V)$; (see [11, [15). Since $H$ is a simple Lie subgroup of $\mathcal{A}^{0}$ it follows that $H$ is contained in $S$. We shall show that $S=H$. First let $C$ be the maximal compact connected normal subgroup of $S$. Since $S$ is semisimple, there exists a closed connected normal subgroup $S^{\prime}$ such that $S=S^{\prime} C$ and $S^{\prime} \cap C$ is a finite subgroup contained in the center of $S$; both $S^{\prime}$ and $C$ being connected subgroups, this implies in particular that every element of $S^{\prime}$ commutes with every element of $C$. Since $H$ is a noncompact connected simple Lie group, it has no nontrivial homomorphism into a compact Lie group. Applying this to the quotient homomorphism of $S$ onto $S / S^{\prime}=C /\left(C \cap S^{\prime}\right)$, we conclude that $H$ is contained 
in $S^{\prime}$. Therefore $C$ is contained in the centralizer of $D$. Since the centralizer is diagonalizable (over the reals) and $C$ is a compact connected subgroup, it follows that $C$ is trivial. Thus $S$ has no compact normal subgroups of positive dimension.

Now recall that the subspace $W$ of $\wedge^{2} V$ is invariant under the action of $\mathcal{A}$ and in particular that of $S$, and let $\varphi: S \rightarrow G L(W)$ be the representation of $S$ induced by the action. Let $K$ be the connected component of the identity in the kernel of $\varphi$. Then $K$ is a connected normal subgroup of $S$. Let $A$ be a maximal subgroup of $S$ such that $D$ is contained in it, and its adjoint action on the Lie algebra of $S$ is diagonalizable over $\mathbb{R}$. Then $A$ intersects (by Iwasawa decomposition) any noncompact connected normal subgroup nontrivially, and since $S$ has no compact normal subgroups, it follows that $A \cap K$ is nontrivial, unless $K$ is trivial. Let $\alpha \in A \cap K$. Then $\alpha$ commutes with all elements of $D$, and hence $\sigma_{1}, \ldots, \sigma_{5}$ are eigenvectors of $\alpha$; let $\lambda_{1}, \ldots, \lambda_{5}$ be the corresponding eigenvalues. Since $\alpha \in K$, the action induced by $\alpha$ on $W$ is trivial. This means that the vectors $\sigma_{1} \wedge \sigma_{4}-\sigma_{2} \wedge \sigma_{3}$, $\sigma_{1} \wedge \sigma_{5}-\sigma_{2} \wedge \sigma_{4}$ and $\sigma_{2} \wedge \sigma_{5}-\sigma_{3} \wedge \sigma_{4}$ are all fixed under the action of $\alpha$. Each $\sigma_{i} \wedge \sigma_{j}$ is an eigenvector of the action of $\alpha$ on $\wedge^{2} V$, and hence the three vectors as above being fixed implies that the vectors $\sigma_{1} \wedge \sigma_{4}, \sigma_{1} \wedge \sigma_{5}, \sigma_{2} \wedge \sigma_{3}, \sigma_{2} \wedge \sigma_{4}$, $\sigma_{2} \wedge \sigma_{5}$ and $\sigma_{3} \wedge \sigma_{4}$ are fixed individually. Therefore for the eigenvalues we get the relations $\lambda_{1} \lambda_{4}=\lambda_{1} \lambda_{5}=\lambda_{2} \lambda_{3}=\lambda_{2} \lambda_{4}=\lambda_{2} \lambda_{5}=\lambda_{3} \lambda_{4}=1$, which is possible only if $\lambda_{i}=1$ for all $i=1, \ldots, 5$. Since $\alpha$ is diagonalizable, this shows that $\alpha$ is the identity. Thus $A \cap K$ is trivial, and hence, as noted above, $K$ is trivial. Therefore the kernel of $\varphi$ is discrete.

Clearly $\varphi(S)$ is a connected semisimple Lie subgroup of $G L(W)$ containing $\varphi(H)$. Since $W$ is 3-dimensional, this implies that $\varphi(S)$ is either $\varphi(H)$ or $S L(W)$. (Inspection of the weights for the action of $\varphi(D)$ on the Lie algebra of $S L(W)$, via the adjoint representation of $S L(W)$, shows that the $\varphi(H)$-action has only two irreducible components, one of them being the Lie subalgebra of $\varphi(H)$ itself. This means that there is not even a subspace invariant under the $\varphi(H)$-action lying strictly between the Lie algebras of $\varphi(H)$ and $S L(W)$, which in particular implies the assertion here; the assertion can also be proved in other ways.) Since $\varphi$ has discrete kernel, this shows that the Lie algebra of $S$ is isomorphic to that of either $H$ or $S L(3, \mathbb{R})$. We note that the Lie algebra of $S L(3, \mathbb{R})$ has no irreducible 5-dimensional representation; this can be deduced from the corresponding statement over the field of complex numbers, the latter being easy to see from the classification theory of representations of semisimple Lie algebras; see [14. Recall that the $H$-action on $V$ is irreducible. Since $S$ contains $H$, its action on $V$ is irreducible, and since $V$ is 5 -dimensional, the preceding observation implies that the Lie algebra of $S$ cannot be isomorphic to that of $S L(3, \mathbb{R})$. Therefore from the alternatives above we now get that $S$ is locally isomorphic to $H$. Since $S$ is connected and $H$ is a subgroup of $S$, this implies that $S=H$.

Since $H=S=\left[\mathcal{A}^{0}, \mathcal{A}^{0}\right]$, in particular $H$ is normal in $\mathcal{A}$. Now let $\alpha \in \mathcal{A}$ be arbitrary, and consider the automorphism of $H$ induced by the conjugation action of $\alpha$. Since $H$ is Lie isomorphic to $P S L(2, \mathbb{R})$, every automorphism of $H$ is inner. Hence there exists $h_{0} \in H$ such that $\alpha h \alpha^{-1}=h_{0} h h_{0}^{-1}$ for all $h \in H$. Then $h_{0}^{-1} \alpha$ commutes with every element of $H$, and in particular with all elements of $D$. Therefore $h_{0}^{-1} \alpha$ is diagonalizable, and in particular all its eigenvalues are real. Since the action of $H$ on $V$ is irreducible, it now follows that $h_{0}^{-1} \alpha$ acts by scalar multiplication by a (nonzero) real number. Thus $h_{0}^{-1} \alpha \in Z$ in the notation of the 
hypothesis. Then $\alpha \in H Z=Z H$, and since $\alpha \in \mathcal{A}$ was arbitrary, we get that $\mathcal{A}$ is contained in $Z H$. On the other hand, $H$ and $Z$ are contained in $\mathcal{A}$, and therefore we have $\mathcal{A}=Z H$. This proves the first assertion in the theorem. The second assertion follows from the fact that $Z H$ is 4 -dimensional while $V$ is 5 -dimensional.

We note also the following simple fact about representations of $P S L(2, \mathbb{R})$ :

Lemma 2.2. Let $\rho: P S L(2, \mathbb{R}) \rightarrow G L(U)$ be a representation of $P S L(2, \mathbb{R})$ over an $\mathbb{R}$-vector space $U$ of dimension $n \geq 5$, such that no nonzero point of $U$ is fixed by the image of $\rho$. Let $E$ be the set of points $p$ in $U$ such that $p$ is an eigenvector of $\rho(g)$ for some nontrivial $g \in P S L(2, \mathbb{R})$. Then $E$ is a union of countably many smooth submanifolds of dimension at most $(n+4) / 2$. In particular, if $L$ is a subspace of $U$ of dimension $m>(n+4) / 2$ and $\lambda$ is the Lebesgue measure on $L$, then $\lambda(E \cap L)=0$.

Proof. We realize $\operatorname{PSL}(2, \mathbb{R})$ as the group $H$ as above (this is just for notational convenience). For each $t \in \mathbb{R}$, let $d_{t}=\exp t \delta$ and $u_{t}=\exp t \nu^{+}$, where $\delta$ and $\nu^{+}$are the matrices defined earlier. Let $\left\{k_{t}\right\}$ be a periodic one-parameter subgroup of $H$, say with period $2 \pi$. Then every element of $H$ is conjugate to one of $d_{t}, u_{t}$ or $k_{t}$ for some $t \in \mathbb{R}$. Let $R$ be the countable subset of $H$ consisting of $d_{1}, u_{1}, k_{1}$ and $k_{r}$ for all $r$ of the form $2 \pi / m$ with $m$ a positive integer.

For any $h \in H$, let $E(h)$ denote the set of points in $U$ that are eigenvectors of $\rho(h)$. Then $E(h)$ is a union of finitely many vector subspaces of $U$; we shall denote by $d(h)$ the maximum of the dimensions of the subspaces contained in $E(h)$. Now consider any nontrivial element $h$ in $H$. We can find a conjugate $h^{\prime}$ of $h$ such that the subgroup generated by $h$ and $h^{\prime}$ is Zariski-dense in $H=P S L(2, \mathbb{R})$. Then every point of $E(h) \cap E\left(h^{\prime}\right)$ is an eigenvector of all elements of $\rho(H)$, and since the latter is a simple Lie group, the point must be fixed by $\rho(H)$. The condition in the hypothesis therefore implies that $E(h) \cap E\left(h^{\prime}\right)=0$. Since $h$ and $h^{\prime}$ are conjugates, we have $d(h)=d\left(h^{\prime}\right)$, and so the preceding conclusion shows that $d(h) \leq n / 2$.

Now let $p \in E$ and $h \in H$ be such that $p$ is an eigenvector of $\rho(h)$. Suppose first that $h$ is of infinite order. There exist $t \in \mathbb{R}$ and $g \in H$ such that $g h g^{-1}$ is one of $d_{t}, u_{t}$ or $k_{t}$. Then $\rho(g)(p)$ is an eigenvector of $d_{t}, u_{t}$ or $k_{t}$. This implies that it is an eigenvector of one of the elements $d_{1}, u_{1}$ or $k_{1}$ respectively; in respect of the last alternative note that, since $k_{t}$ is of infinite order, the closure of the cyclic subgroup generated by it contains $k_{1}$. Thus there exists $x \in R$ such that $\rho(g)(p) \in E(x)$, and so $p \in \rho(H) E(x)$. Now suppose that $h$ as above is of finite order. Then $h$ is conjugate to $k_{r}$ for some $r$ of the form $2 \pi / m$ for a positive integer $m$. Therefore in this case also we get that $\rho(g)(p) \in E(x)$ for some $x \in R$. Thus $E=\bigcup_{x \in R} \rho(H)(E(x))$. Each $E(x)$ is a finite union of vector subspaces that are invariant under a one-parameter subgroup of $H$ and have dimension at most $n / 2$. It follows that each $\rho(H)(E(x))$ is a finite union of smooth submanifolds of dimension at most $(n / 2)+2=(n+4) / 2$. This proves the lemma.

With respect to the conclusion of the lemma, the situation in low dimensions is as follows. For dimensions 2 and 4 there are no representations of $\operatorname{PSL}(2, \mathbb{R})$ with no nonzero common fixed points. In dimension 3 the realization of $H$ (as above) as a subgroup of $S L(3, \mathbb{R})$ is (up to equivalence) the only representation with no common fixed points. For this representation the set of points that are eigenvectors of nontrivial elements forms a cone in $\mathbb{R}^{3}$ with nonempty interior. 


\section{A 2-STEP NiLPotent Lie Group}

We now use Theorem 2.1 to give an example of a simply connected 2-step nilpotent Lie group $G$ such that the action of $\operatorname{Aut}(G)$ on $G$ has no dense orbit.

Let $V$ and $W \subset \wedge^{2} V$ be as in the preceding section and let $\mathcal{G}$ be the 2-step nilpotent Lie algebra associated with the pair $V, W$, as in $\S$; namely $\mathcal{G}=V \oplus$ $V^{\prime}$, where $V^{\prime}=\left(\wedge^{2} V\right) / W$ and the Lie bracket operation is determined by the conditions $\left[v_{1}, v_{2}\right]=\left(v_{1} \wedge v_{2}\right)(\bmod W)$ for all $v_{1}, v_{2} \in V$, and $[x, y]=0$ for $x \in \mathcal{G}$ and $y \in V^{\prime}$. Let $G$ be the simply connected nilpotent Lie group corresponding to $\mathcal{G}$. Then we have

Corollary 3.1. The action of Aut $(G)$ on $G$ has no dense orbit.

Proof. For the Lie algebra $\mathcal{G}$ as above, corresponding to $G$, the subgroup $\mathcal{A}(\mathcal{G})$ defined in $\S \square$ is the same as the subgroup $\mathcal{A}$ in Theorem 2.1 Since by Theorem [2.1] the action of $\mathcal{A}$ on $V$ has no open orbit, by Proposition 1.2 the Aut $(G)$-action on $G$ has no dense orbit.

Using Theorem 2.1 one can also describe $\operatorname{Aut}(G)$ as follows. To each $h \in H$ there corresponds a Lie automorphism of $\mathcal{G}$ such that the restrictions to $V$ and $V^{\prime}=\left(\wedge^{2} V\right) / W$ are respectively given by the $h$-action on $V$ and the factor of the $h$-action on $\wedge^{2} V$. We denote by $\tilde{H}$ the group of automorphisms of $G$ for which the associated Lie automorphism corresponds to some $h \in H$ as above. Then $\tilde{H}$ is a Lie subgroup of Aut $(G)$, canonically Lie isomorphic to $H$. Similarly each $z \in Z$ corresponds canonically to an automorphism of $G$, and we shall denote by $\tilde{Z}$ the subgroup of Aut $(G)$ corresponding to $Z$. We note that the center of $\mathcal{G}$ contains $V^{\prime}=[\mathcal{G}, \mathcal{G}]$, and since the $H$-action on $V$ is irreducible, it coincides with $[\mathcal{G}, \mathcal{G}]$; hence $[G, G]$ is the center of $G$. Let $\Psi$ be the group of shear automorphisms of $G$ (see $\S$ 1). Then $\Psi$ consists precisely of $\tau \in \operatorname{Aut}(G)$ such that $\tau(g) \in g[G, G]$ for all $g \in G$.

Corollary 3.2. Aut $(G)=(\tilde{Z} \tilde{H}) \Psi$, a semidirect product (with $\Psi$ as the normal subgroup).

Proof. We note that $(\tilde{Z} \tilde{H}) \Psi$ is a subgroup of Aut $(G)$. Now consider the homomorphism $\tau \mapsto \bar{\tau}$ of Aut $(G)$ into $G L(V)$. Its kernel is $\Psi$, and its image is $\mathcal{A}$, which by Theorem 2.1 is $Z H$. Since the subgroup $(\tilde{Z} \tilde{H}) \Psi$ of Aut $(G)$ contains $\Psi$, and its image under the homomorphism as above is $Z H$, this shows that it must be the whole. Since the map $\tau \mapsto \bar{\tau}$ is injective on $\tilde{Z} \tilde{H}$, it follows that $(\tilde{Z} \tilde{H}) \cap \Psi$ is trivial. This proves the corollary.

Remark 3.3. The action of $H$ on $\wedge^{2} V$ (notation as before) decomposes into two irreducible components, one being on the subspace $W$ as above and the other on the subspace, say $W^{\prime}$, spanned by the $H$-orbit of $\sigma_{1} \wedge \sigma_{2}$; this can be deduced by inspection of the set of weights. Let $\mathcal{G}^{\prime}$ be the 2-step nilpotent Lie algebra associated with the pair $V$ and $W^{\prime}$, as described in $\S$, and let $G^{\prime}$ be the simply connected Lie group with Lie algebra $\mathcal{G}^{\prime}$. Then with some modifications in the above argument it can be shown that $\operatorname{Aut}\left(G^{\prime}\right)$ has no open orbit on $G^{\prime}$; in this respect we note mainly that $W$ is also invariant, along with $W^{\prime}$, under the action of $\mathcal{A}\left(G^{\prime}\right)$ on $\wedge^{2} V$. This yields an 8-dimensional example (as against 12 of $G$ ) for which the action of the automorphism group has no dense orbit. The example in Theorem 2.1, on the other hand, admits a simpler presentation. 


\section{LIE GROUPS WITH NILPOTENT AUTOMORPHISM GROUPS}

An example of a 6-step simply connected nilpotent Lie group $G$ such that Aut $(G)$ is nilpotent was given in [10]. Earlier in [9] an example of a 3-step nilpotent Lie algebra $\mathcal{G}$ was constructed for which all derivations are nilpotent; the condition implies that Aut $(\mathcal{G})^{0}$, the connected component of the identity in $\operatorname{Aut}(\mathcal{G})$, consists of unipotent elements (viewed as elements in $G L(\mathcal{G})$ ), and hence it is a nilpotent Lie group. Aut $(\mathcal{G})$ itself is however not nilpotent for that example, as has been remarked in [10]. In this section we shall describe a class of examples, of simply connected 3-step nilpotent Lie groups, related to the results in the preceding sections, for which all the automorphisms are unipotent, and the automorphism groups are nilpotent. For a subclass of the examples, the automorphism groups are 2-step nilpotent Lie groups with one-dimensional commutator subgroup. The following remarks highlight the significance of examples with these properties.

Remark 4.1. Let $\mathcal{G}$ be a nilpotent Lie algebra such that Aut $(\mathcal{G})^{0}$ consists of unipotent elements. Then Aut $(\mathcal{G})^{0}$ is a unipotent algebraic subgroup of $G L(\mathcal{G})$, and hence all its orbits on $\mathcal{G}$ are closed (see [11]). Since Aut $(\mathcal{G})$ is an algebraic group, it has only finitely many connected components, and hence we get furthermore that all orbits of Aut $(\mathcal{G})$ on $\mathcal{G}$ are closed. Since the Aut $(G)$-action on $G$ and the Aut $(\mathcal{G})$ action on $\mathcal{G}$ are topologically equivalent (via the exponential map), it follows that all orbits of Aut $(G)$ on $G$ are closed; note that they have to be proper closed subsets. This establishes a stronger property than the orbits being non-dense, which was obtained in $\S 3$

The next remark shows optimality of the 3-step condition with respect to nilpotent Lie groups $G$ such that all Aut $(G)$-orbits on $G$ are closed.

Remark 4.2. Let $G$ be a 2 -step simply connected nilpotent Lie group. Then the group Aut $(G)^{0}$ is not nilpotent, and for the action of Aut $(G)$ on $G$, the closure of every orbit contains the identity element in $G$ (in particular, orbits other than that of the identity element are not closed). This can be seen as follows. Let $\mathcal{G}$ be the Lie algebra of $G$. We realize $\mathcal{G}$ as $V \oplus\left(\wedge^{2} V\right) / W$, where $V=\mathcal{G} /[\mathcal{G}, \mathcal{G}]$ and $W$ is a subspace of $\wedge^{2} V$ (see $\S \mathbb{1}$ ). For $t \in \mathbb{R}^{+}$, let $\varphi_{t}: \mathcal{G} \rightarrow \mathcal{G}$ be the linear map such that $\varphi_{t}(v)=t v$ for all $v \in V$ and $\varphi_{t}\left(v^{\prime}\right)=t^{2} v^{\prime}$ for all $v^{\prime} \in \wedge^{2} V$. Then $\left\{\varphi_{t}\right\}$ is in fact a one-parameter subgroup of $\operatorname{Aut}(\mathcal{G})$. We shall denote the corresponding oneparameter subgroup of Aut $(G)$ also by $\left\{\varphi_{t}\right\}$. Let $G^{*}$ be the subgroup of Aut $(G)$ consisting of all inner automorphisms of $G$. Then $G^{*}$ is canonically isomorphic to a nontrivial quotient of $V$ by a vector subspace, and the conjugation action of $\varphi_{t}$ on $G^{*}$ in Aut $(G)$ corresponds to scalar multiplication by $t$. It follows that the subgroup generated by $\left\{\varphi_{t}\right\}$ and $G^{*}$ in Aut $(G)$ is not nilpotent. Hence Aut $(G)^{0}$ is not nilpotent. Also, for the action of $\left\{\varphi_{t}\right\}$ on $\mathcal{G}$ the zero element is in the closure of every orbit, and hence under the $\left\{\varphi_{t}\right\}$-action on $G$ the identity element is in the closure of every orbit (this also shows, in view of Remark 4.1 that Aut $(G)^{0}$ cannot be nilpotent).

Remark 4.3. For any simply connected Lie group $G$ (not necessarily nilpotent) of dimension at least 2 , Aut $(G)^{0}$ is nonabelian. This is clear for vector groups, and Remark 4.2 shows that it holds for 2-step nilpotent Lie groups. If $G$ is neither abelian nor a 2-step nilpotent Lie group, then the group of inner automorphisms is nonabelian, and hence so is Aut $(G)^{0}$. Thus for any simply connected Lie group 
$G$ the group [Aut $(G)^{0}$, Aut $\left.(G)^{0}\right]$ is of dimension at least 1 , and for the examples in Corollary 4.5 below the minimum is attained. [In this context it may be mentioned that by an analogous argument it can be shown that for any connected (not necessarily simply connected) Lie group $G$ other than the circle group, the automorphism group Aut $(G)$ is nonabelian; if $G$ is not assumed to be simply connected, then Aut $(G)^{0}$ can however be trivial, as in the case of tori, or a nontrivial abelian group as in the case of the examples in Corollary 5.2 in the next section.]

We now proceed to describe the 3 -step nilpotent Lie algebras for our examples. We shall follow the notation of $\S\left[2\right.$. For $1 \leq i<j \leq 5$, let $p_{i j}=\sigma_{i} \wedge$ $\sigma_{j} \bmod (W)$. We note that $p_{14}=p_{23}, p_{15}=p_{24}$, and $p_{25}=p_{34}$, and that $\left\{p_{12}, p_{13}, p_{14}, p_{15}, p_{25}, p_{35}, p_{45}\right\}$ is a basis of $V^{\prime}$. Let $L$ be the subspace of $V^{\prime}$ spanned by $\left\{p_{13}, p_{14}, p_{15}, p_{25}, p_{35}, p_{45}\right\}$. Then by Lemma 2.2 there exists (a nonzero element) $p \in L$ which is not an eigenvector of the action of any nontrivial element of $H$ (under the $H$-action on $V^{\prime}$ ). Now we set $\mathcal{N}=V \oplus V^{\prime}$ and define a Lie bracket operation by the relations $\left[\sigma_{i}, \sigma_{j}\right]=\sigma_{i} \wedge \sigma_{j}(\bmod W)$ for all $i, j=1, \ldots, 5,\left[x, x^{\prime}\right]=0$ for all $x, x^{\prime} \in V^{\prime},\left[\sigma_{1}, p_{12}\right]=p$, and $\left[\sigma_{k}, p_{i j}\right]=0$ if either $k \neq 1$ or $(i, j) \neq(1,2)$; the product defined on the basis elements extends uniquely to a Lie bracket operation on $\mathcal{N}$; we note in particular that by definition $\left[\sigma_{i},\left[\sigma_{j}, \sigma_{k}\right]\right]=0$ if $i, j$ and $k$ are distinct indices, from which one can see that the Jacobi identity holds for the product. Furthermore, since $p$ is contained in the above subspace $L$, it follows that $p$ is contained in the center of $\mathcal{N}$. Since $p$ spans $[\mathcal{N},[\mathcal{N}, \mathcal{N}]]$ this shows that $\mathcal{N}$ is a 3 -step nilpotent Lie algebra. Let $N$ be the simply connected (nilpotent) Lie group corresponding to $\mathcal{N}$.

We prove the following:

Corollary 4.4. The subgroup $\mathcal{A}(N)$ consists only of the identity element. Consequently, Aut $(N)$ consists of unipotent elements (when realized canonically as a subgroup of $G L(\mathcal{N})$ ), and hence it is a simply connected nilpotent Lie group.

Proof. Let $\tau \in \operatorname{Aut}(\mathcal{N})$. Then $\tau$ factors to a linear transformation $x=\bar{\tau} \in G L(V)$. Since $\left[\sigma_{i}, \sigma_{j}\right]=\sigma_{i} \wedge \sigma_{j}(\bmod W)$ for all $i, j=1, \ldots, 5$, it follows that the action of $x$ on $\wedge^{2} V$ leaves invariant the subspace $W$. Hence by Theorem 2.1, $x \in Z H$. Let $z \in Z$ and $h \in H$ be such that $x=z h$. Since the subspace spanned by $p$ equals $[\mathcal{N},[\mathcal{N}, \mathcal{N}]]$, it is invariant under all automorphisms of $\mathcal{N}$. Thus $p$ is an eigenvector of $x$ (for the action on $V^{\prime}$ ). Since the elements of $Z$ act as scalars on $V^{\prime}$, it follows that $p$ is an eigenvector of $h$. Since by choice $p$ is not an eigenvector of any nontrivial element of $H$, we get that $h$ is trivial. Thus $\bar{\tau}=x=z$, a scalar transformation, say multiplication by $\lambda$. Then the action of $\tau$ on $V^{\prime}$ is given by multiplication by $\lambda^{2}$. Since $\left[\sigma_{1}, p_{12}\right]=p$, we have $\lambda^{2} p=\tau(p)=\tau\left(\left[\sigma_{1}, p_{12}\right]\right)=$ $\left[\tau\left(\sigma_{1}\right), \tau\left(p_{12}\right)\right]=\lambda^{3}\left[\sigma_{1}, p_{12}\right]=\lambda^{3} p$. Since $p$ is a nonzero element, this implies that $\lambda=1$. This means that $z$ is trivial, and therefore $\bar{\tau}$ is trivial. Thus $\bar{\tau}$ is trivial for all $\tau \in \operatorname{Aut}(\mathcal{N})$. This shows that $\mathcal{A}(N)$ is trivial. The second assertion follows from this, together with Lemma 1.1 and the fact that every algebraic subgroup consisting of unipotent elements is simply connected (see [15], Theorem 3.6.3). This proves the corollary.

We deduce also the following, showing that under a slight further condition, the automorphism group is "minimum possible", since it admits only the mandatory automorphisms. 
Corollary 4.5. Let $N$ and $\mathcal{N}$ be as above. Suppose that the point $p$ in the definition of the Lie algebra structure on $\mathcal{N}$ is not contained in the subspace spanned by $\left\{p_{14}, p_{15}, p_{25}, p_{35}, p_{45}\right\}$. Let $N^{*}$ denote the subgroup of Aut $(N)$ consisting of all inner automorphisms, and let $\Psi$ be the subgroup consisting of all shear automorphisms of $N$. Then Aut $(N)=N^{*} \Psi$. Furthermore, Aut $(N)$ is a simply connected 2-step nilpotent Lie group and $[\operatorname{Aut}(N)$, Aut $(N)]$ is one-dimensional.

Proof. We shall identify Aut $(N)$ with $\operatorname{Aut}(\mathcal{N})$ as before. As an algebraic subgroup consisting of unipotent elements, Aut $(\mathcal{N})$ is a connected Lie group. The Lie algebra $\mathcal{D}$ consisting of all derivations of $\mathcal{N}$ is the Lie algebra of Aut $(\mathcal{N})$. The subgroups $N^{*}$ and $\Psi$ are normal Lie subgroups of $\operatorname{Aut}(\mathcal{N})$. Therefore to prove the corollary it suffices to show that the Lie subalgebras of $\mathcal{D}$ corresponding to $N^{*}$ and $\Psi$ span $\mathcal{D}$. The Lie subalgebra corresponding to $\Psi$ consists of all derivations $\delta$ such that $\delta(\mathcal{N})$ is contained in $L$. We shall show that this Lie subalgebra and ad $\sigma_{1}$ and ad $\sigma_{2}$ together span $\mathcal{D}$ as a vector space. This would complete the proof.

Let $\delta$ be any derivation of $\mathcal{N}$. In view of Corollary 4.4, its factor on $\mathcal{N} /[\mathcal{N}, \mathcal{N}]$ is trivial, and hence $\delta(\mathcal{N})$ is contained in $V^{\prime}$. Hence there exist $a_{1}, a_{2} \in \mathbb{R}$ such that $\delta\left(\sigma_{i}\right) \in a_{i} p_{12}+L, i=1,2$. Then $\delta^{\prime}=\delta-a_{2}\left(\operatorname{ad} \sigma_{1}\right)+a_{1}\left(\operatorname{ad} \sigma_{2}\right)$ is a derivation such that $\delta^{\prime}\left(\sigma_{1}\right), \delta^{\prime}\left(\sigma_{2}\right) \in L$. It suffices to show that $\delta^{\prime}$ belongs to the Lie subalgebra of $\Psi$, namely that $\delta^{\prime}(\mathcal{N})$ is contained in $L$. Changing notation, we shall assume that $\delta\left(\sigma_{1}\right), \delta\left(\sigma_{2}\right) \in L$ and deduce that $\delta(\mathcal{N}) \subset L$.

Since $\delta(\mathcal{N}) \subset V^{\prime}$, for $i, j \geq 2$ we have $\delta\left(p_{i j}\right)=\delta\left(\left[\sigma_{i}, \sigma_{j}\right]\right)=\left[\delta \sigma_{i}, \sigma_{j}\right]+\left[\sigma_{i}, \delta \sigma_{j}\right]=$ 0 . Since $p_{14}=p_{23}$ and $p_{15}=p_{24}$, it also follows that $\delta\left(p_{14}\right)=\delta\left(p_{15}\right)=0$. We note that $\delta\left(p_{12}\right)=\delta\left(\left[\sigma_{1}, \sigma_{2}\right]\right)=\left[\delta \sigma_{1}, \sigma_{2}\right]+\left[\sigma_{1}, \delta \sigma_{2}\right] \in[\mathcal{N},[\mathcal{N}, \mathcal{N}]] \subset L$. Therefore $\delta(p)=\delta\left(\left[\sigma_{1}, p_{12}\right]\right)=\left[\delta \sigma_{1}, p_{12}\right]+\left[\sigma_{1}, \delta p_{12}\right]=0$. Let $L^{\prime}$ be the subspace spanned by $\left\{p_{14}, p_{15}, p_{25}, p_{35}, p_{45}\right\}$. Then, from what we have seen, $\delta(x)=0$ for all $x \in L^{\prime}$. By the assumption on $p$ there exists $\lambda \neq 0$ such that $p=\lambda p_{13}+x$ for some $x \in L^{\prime}$. Since $\delta(p)=0$ and $\delta(x)=0$, this implies that $\delta\left(p_{13}\right)=0$. Thus we have $\left[\sigma_{1}, \delta\left(\sigma_{j}\right)\right]=\delta\left(\left[\sigma_{1}, \sigma_{j}\right]\right)=\delta\left(p_{1 j}\right)=0$ for $j=3,4,5$. This shows that $\delta\left(\sigma_{j}\right) \in L$ for $j=3,4,5$. Since by assumption $\delta\left(\sigma_{1}\right), \delta\left(\sigma_{2}\right) \in L$, we now have $\delta\left(\sigma_{i}\right) \in L$ for all $i=1, \ldots, 5$. Therefore $\delta(\mathcal{N})$ is contained in $L$. As noted above, this shows that Aut $(N)=N^{*} \Psi$.

The Lie algebra of $N^{*}$ is canonically isomorphic to $\mathcal{N} / L$. Also we clearly have $[\mathcal{N} / L, \mathcal{N} / L]=V^{\prime} / L$, which is one-dimensional, and therefore $\left[N^{*}, N^{*}\right]$ is onedimensional. It is straightforward to verify that the automorphisms from $N^{*}$ and $\Psi$ commute with each other. Since Aut $(N)=N^{*} \Psi$ and $\Psi$ is abelian, this implies that $\operatorname{Aut}(N)$ is a simply connected nilpotent Lie group and [Aut $(N)$, Aut $(N)$ ] is one-dimensional; in view of the latter condition, Aut $(N)$ is in fact a 2-step simply connected nilpotent Lie group. This completes the proof.

\section{NON-SIMPLY-CONNECTED LiE GROUPS}

In this section we shall discuss the analogous questions for Lie groups which are not necessarily simply connected. We begin with the following observation.

Proposition 5.1. Let $G$ be a connected abelian Lie group. Then the action of Aut $(G)$ on $G$ has a dense orbit if and only if $G$ is not (topologically isomorphic to) the circle group.

Proof. It is well known that for $m \geq 2$ the $m$-dimensional torus admits automorphisms with dense orbits (see [16], for instance). Now let $G=V \times C$, where $V$ is 
a vector space of dimension $n \geq 1$ and $C$ is the torus of dimension $m \geq 0$. Let $\mathcal{H}$ be the set of all continuous homomorphisms of $V$ into $C$. For each $\tau \in G L(V)$ and $\psi \in \mathcal{H}$ we get a continuous automorphism of $G$ defined by $(v, c) \mapsto(\tau(v), c \psi(v))$ for all $v \in V$ and $c \in C$. The automorphisms arising in this way form a subgroup of Aut $(G)$ (it is in fact the identity component of the latter). We recall that there exist homomorphisms $\psi: V \rightarrow C$ such that $\psi(V)$ is dense in $C$. Using this, it is straightforward to verify that under the action of the group of automorphisms as above the orbit of any element of the form $(v, c)$ with $v \neq 0$ is dense in $G$. Thus the Aut $(G)$-action on $G$ has dense orbits. Finally, the circle group has only two automorphisms, and hence in this case the Aut $(G)$-action has no dense orbit. This proves the proposition.

For Lie groups $G$ of the form $\mathbb{R}^{n} \times \mathbb{T}^{m}$ with $m \neq 1$ there exist abelian subgroups of $\operatorname{Aut}(G)$, with finite rank, whose action on $G$ has dense orbits; see [5] for more precise results in this respect.

In the context of the results in the last section and Proposition 5.1, one may ask whether there exist connected 2-step nilpotent Lie groups $G$ such that all orbits of the Aut $(G)$-action on $G$ are closed. By Remark 4.2 such a group is necessarily not simply connected. The following corollary shows that there are groups with this property among quotients of the group $G$ of $\S 3$, by discrete central subgroups.

Corollary 5.2. Let $G$ be the simply connected 2-step nilpotent Lie group of Corollary 3.1. Then there exists a subset of $E$ of Lebesgue measure 0 in $[G, G]$ such that if $\theta \in[G, G], \theta \notin E$ and $\Theta$ is the cyclic subgroup of $G$ generated by $\theta$, then for the Lie group $G^{\prime}=G / \Theta$ the following holds: $\left[G^{\prime}, G^{\prime}\right]$ is a closed subgroup of $G^{\prime}$ and for any automorphism $\tau$ of $G^{\prime}$ the factor of $\tau$ on $G^{\prime} /\left[G^{\prime}, G^{\prime}\right]$ is $\pm I$, where $I$ is the identity transformation; furthermore, orbits of the action of $\operatorname{Aut}\left(G^{\prime}\right)$ on $G^{\prime}$ consist of either one or two cosets of $\left[G^{\prime}, G^{\prime}\right]$ in $G^{\prime}$.

Proof. Let $\tilde{H}$ be the group of automorphisms in 9 , and consider the $\tilde{H}$-action on $[G, G]$. Recall that the latter may be viewed as a vector space and that the action is linear and irreducible; in particular there is no nonzero point fixed by the whole of $\tilde{H}$. Since $\tilde{H}$ is Lie isomorphic to $\operatorname{PSL}(2, \mathbb{R})$ and $[G, G]$ is 7-dimensional, by Lemma 2.2 there exists a subset $E$ of Lebesgue measure 0 such that points outside $E$ are not eigenvectors of any nontrivial element of $\tilde{H}$. Now let $\theta \in[G, G], \theta \notin E$, and let $\Theta$ be the cyclic subgroup generated by $\theta$; since $[G, G]$ is central in $G, \Theta$ is a normal subgroup. Let $G^{\prime}=G / \Theta$. Since $\Theta$ is discrete, the automorphisms of $G^{\prime}$ are precisely factors on $G^{\prime}$ of automorphisms $\tau$ of $G$ such that $\tau(\Theta)=\Theta$. We note that the shear automorphisms, namely those from $\Psi$ in the notation of 43 , satisfy the condition. Therefore by Corollary 3.2, Aut $\left(G^{\prime}\right)$ is the semidirect product of $\Psi$ with the subgroup $\{\tau \in \tilde{Z} \tilde{H} \mid \tau(\Theta)=\Theta\}$. Now let $\tau=z h \in \tilde{Z} \tilde{H}$, where $z \in \tilde{Z}$ and $h \in \tilde{H}$, be such that $\tau(\Theta)=\Theta$. Since $z$ acts by scalar multiplication on $[G, G]$, this implies that $\theta$ is an eigenvector of $h$. Since $\theta \notin E$, it follows that $h$ is the identity element. Hence $\tau=z \in \tilde{Z}$, and since $\tau(\Theta)=\Theta$, it follows that the corresponding scalar transformation of $V=G /[G, G]$ is $\pm I$, where $I$ denotes the identity transformation. Clearly $\left[G^{\prime}, G^{\prime}\right]=[G, G] / \Theta$, which is a closed subgroup of $G^{\prime}=G / \Theta$, and hence the preceding conclusion implies that the factor of any $\tau^{\prime} \in \operatorname{Aut}\left(G^{\prime}\right)$ on $G^{\prime} /\left[G^{\prime}, G^{\prime}\right]$ is $\pm I$. This proves the first assertion in the corollary. The second one follows from this, together with the fact that automorphisms from $\Psi$ factor to $G^{\prime}$. 


\section{Anosov automorphisms}

As mentioned in the introduction, study of the automorphism group can be applied also to the general question of understanding the class of compact nilmanifolds supporting Anosov automorphisms; see [1], 3], 7]. In this respect we note the following consequence of Theorem 2.1.

Corollary 6.1. Let $G$ be the 2-step simply connected nilpotent Lie group of Theorem 2.1, and let $V=G /[G, G]$ (as before, realized as a vector space). If $\tau \in$ Aut $(G)$ and the factor $\bar{\tau}$ on $V$ has determinant 1 , then $\bar{\tau}$ has a nonzero fixed point on $V$. Consequently, if $\Gamma$ is a lattice (a discrete cocompact subgroup) in $G$ and $\tau \in$ Aut $(G)$ is such that $\tau(\Gamma)=\Gamma$, then the factor automorphism $\pi(\tau): G / \Gamma \rightarrow G / \Gamma$ is not an Anosov automorphism, and furthermore it is not ergodic.

Proof. We continue to use our previous notation. By Theorem 2.1, $\bar{\tau} \in Z H$, and furthermore if its determinant is 1 , then $\bar{\tau} \in H$. The first assertion therefore follows from the fact that for the $H$-action on $V$ every element of $h$ has a nonzero fixed point in $V$; for hyperbolic elements this follows from consideration of weights, for parabolic elements by unipotence, and for elliptic elements by odd-dimensionality of $V$ (the analogous assertion holds for any irreducible representation of $H$ on any odd-dimensional vector space).

If $\Gamma$ is a lattice in $G$, then $[G, G] \Gamma$ is closed and $[G, G] \Gamma /[G, G]$ is a lattice in $V=G /[G, G]$; see 13 , Corollary 1 to Theorem 2.3. Therefore for any $\tau \in \operatorname{Aut}(G)$ such that $\tau(\Gamma)=\Gamma, \bar{\tau}$ has determinant \pm 1 . Hence by the first part, $\bar{\tau}^{2}$ has nonzero fixed points in $V$. This implies that the factor of $\tau$ on $G / \Gamma$ is not an Anosov automorphism, and also that it is not ergodic. This proves the corollary.

Compact nilmanifolds covered by the 3-step simply connected nilpotent Lie group $N$ as in $\S 4$ also do not support Anosov automorphisms; this is immediate from the fact that there are no hyperbolic automorphisms since $[N,[N, N]]$ is one-dimensional.

Acknowledgement. The author would like to thank Karel Dekimpe and the referee for useful comments on earlier versions of this paper.

\section{REFERENCES}

[1] L. Auslander and J. Scheuneman, On certain automorphisms of nilpotent Lie groups, Global Analysis (Proc. Sympos. Pure Math., Vol. XIV, Berkeley, Calif. 1968) pp. 9-15, Amer. Math. Soc., Providence, RI, 1970. MR 42:5284

[2] A. Bialynicki-Birula and M. Rosenlicht, Injective morphisms of real algebraic varieties, Proc. Amer. Math. Soc. 13 (1962), 200-203. MR 25:3936

[3] S.G. Dani, Nilmanifolds with Anosov automorphisms, J. London Math. Soc. (2) 18 (1978), 553-559. MR 80k:58082

[4] S.G. Dani, On automorphism groups acting ergodically on connected locally compact groups, Ergodic Theory and Harmonic Analysis (Mumbai, 1999), Sankhya, Ser. A, 62 (2000), 360-366. MR 2001m:22013

[5] S.G. Dani, On ergodic $\mathbb{Z}^{d}$-actions on Lie groups by automorphisms, Israel J. Math. 126 (2001), 327-344. MR 2002j:37006

[6] S.G. Dani and M. McCrudden, A criterion for exponentiality in certain Lie groups, J. Algebra 238 (2001), 82-98. MR 2002b:22010

[7] K. Dekimpe, Hyperbolic automorphisms and Anosov diffeomorphisms on nilmanifolds, Trans. Amer. Math. Soc. 353 (2001), 2859-2877. MR 2002c:37043

[8] K. Dekimpe and W. Malfait, A special class of nilmanifolds admitting an Anosov diffeomorphism, Proc. Amer. Math. Soc. 128 (2000), 2171-2179. MR 2000m:37029 
[9] J. Dixmier and W.G. Lister, Derivations of nilpotent Lie algebras, Proc. Amer. Math. Soc. 8 (1957), 155-158. MR 18:659a

[10] J. L. Dyer, A nilpotent Lie algebra with nilpotent automorphism group, Bull. Amer. Math. Soc. 76 (1970), 52-56. MR 40:2789

[11] G. P. Hochschild, The Basic Theory of Algebraic Groups and Lie Algebras, Graduate Texts in Mathematics 75, Springer-Verlag, 1981. MR 82i:20002

[12] W. Malfait, Anosov diffeomorphisms on nilmanifolds of dimension at most six, Geometriae Dedicata (3) 79 (2000), 291-298. MR 2001h:20041

[13] M.S. Raghunathan, Discrete Subgroups of Lie Groups, Springer-Verlag, 1972. MR 58:22394a

[14] J.-P. Serre, Complex Semisimple Lie Algebras, Springer Monographs in Mathematics, Springer-Verlag, 2001 (reprinted edition). MR 2001h:17001

[15] V.S. Varadarajan, Lie Groups, Lie Algebras and their Representations, Graduate Texts in Mathematics 102, Springer-Verlag, 1984 (reprinted edition). MR 85e:22001

[16] P. Walters, An Introduction to Ergodic Theory, Graduate Texts in Mathematics 79, SpringerVerlag, 1982. MR 84e:28017

Erwin Schrödinger Institute, Boltzmanngasse 9, A-1090 Vienna, Austria

Current address: School of Mathematics, Tata Institute of Fundamental Research, Homi Bhabha Road, Colaba, Mumbai 400 005, India

E-mail address: dani@math.tifr.res.in 